\title{
Funkzellenabfragen auf dem Prüfstand
}

Die Verkehrsdaten der Mobilkommunikation wecken zunehmend das Interesse der Strafverfolgungsbehörden, weil sie nicht nur die Rufnummern des Anrufers und des Angerufenen, sondern auch Standortdaten beider Kommunikationsteilnehmer enthalten Die Strafprozessordnung erlaubt unter engen Voraussetzungen sowohl eine individualisierte als auch eine nicht-individualisierte verdeckte Abfrage von Funkzellendaten.

Bei der praktisch bedeutsameren nicht-individualisierten Funkzellenabfrage müssen ein Verdächtiger und seine Mobilfunknummer dagegen nicht bekannt sein. Sie dient vielmehr der Verdachtsgewinnung und greift deshalb in die Grundrechte einer Vielzahl von unverdächtigen Personen ein, die sich in aller Regel zufällig in der Funkzelle aufhalten. Deshalb hat der Gesetzgeber diese einschneidendere Form der Funkzellenabfrage an noch engere Voraussetzungen geknüpft.

Erstmals standen individualisierte Funkzellenabfragen im Fokus der öffentlichen Aufmerksamkeit, nachdem die sächsischen Strafverfolgungsbehörden im Februar 2011 anlässlich einer Demonstration gegen Neo-Nazis in Dresden eine flächendeckende Funkzellenabfrage durchführten. Der Sächsische Datenschutzbeauftragte prüfte dieses Vorgehen ${ }^{1}$ und stellte gravierende datenschutzrechtliche Mängel fest. Die Konferenz der Datenschutzbeauftragten des Bundes und der Länder hat eine verfehlte Kritik am Sächsischen Datenschutzbeauftragten zurückgewie$\mathrm{sen}^{2}$. Die Tatsache, dass Funkzellenabfragen richterlich genehmigt werden müssen, führt nicht dazu, dass Polizei und Staatsanwaltschaft von der Datenschutzkontrolle freigestellt werden.

Im Januar 2012 wurde bekannt, dass die Berliner Polizei schon seit 2009 im Zusammenhang mit einer Serie von Autobrandstiftungen zum Mittel der nicht-individualisierten Funkzellenabfrage gegriffen hatte, ohne dass dies zur Ergreifung der Täter geführt hätte. Daraufhin überprüfte der Berliner Beauftragte für Datenschutz und Informationsfreiheit die Praxis der Strafverfolgungsbehörden im Zeitraum von 2009 - 2011 und stellte ebenfalls gravierende Verstöße gegen die Strafprozessordnung fest.

Insgesamt wurden im Rahmen einer stichprobenartigen Kontrolle 108 staatsanwaltschaftliche Ermittlungsakten überprüft. Dabei stellte sich heraus, dass Funkzellenabfragen schwerpunktmäßig zur Aufklärung von Brandstiftungen, Betrug, Raub und vergleichbaren Delikte eingesetzt wurden. Lediglich in zwei Betrugsfällen, jedoch in keinem der Fälle von Kfz-Brandstiftung

1 DuD 2011, Heft 9, S.668

2 Entschließung der 82. Konferenz der Datenschutzbeauftragten des Bundes und der Länder am 28./29.9.2011 („Vorbeugender Grundrechtsschutz ist Aufgabe der Datenschutzbeauftragten !"), DuD, 2011, Heft 11, S.812 führte die Funkzellenabfrage zur Ermittlung eines Täters. Auch wenn Kfz-Brandstiftung zu den Katalogstraftaten des $\$ 100 \mathrm{a}$ StPO zählt, bestanden deshalb Zweifel daran, ob Funkzellenabfragen zur Aufklärung solcher Brandstiftungen geeignet waren.

Funkzellenabfragen, die aufgrund ihrer Eingriffsintensität und großen Streubreite nur in Ausnahmefällen durchgeführt werden dürfen, sind in bestimmten Deliktsbereichen offensichtlich zum alltäglichen Ermittlungsinstrument geworden, das routinemäßig und ohne hinreichende Beachtung der gesetzlichen Vorgaben eingesetzt wird. Dieser oft bedenkenlose Einsatz der Maßnahme wird durch die fehlende Pflicht zur Dokumentation der Entscheidungsbegründung über die Durchführung einer Funkzellenabfrage gefördert.

Folgende Empfehlungen hat der Berliner Beauftragte für Datenschutz und Informationsfreiheit ausgesprochen:

- Bezüglich der zurückliegenden Verfahren sind die Betroffenen, deren Bestandsdaten erhoben wurden, zu benachrichtigen und über ihre Rechtsschutzmöglichkeiten aufzuklären. Die erhobenen Daten sind unverzüglich zu löschen, soweit sie nicht nachweislich weiterhin zur Strafverfolgung oder möglichen gerichtlichen Überprüfungen erforderlich sind. Die Löschung und die Begründung für eine weitere Aufbewahrung sind aktenkundig zu machen.

- Für die Zukunft sollte durch Dienstanweisungen festgelegt werden, dass die Begründung für den Einsatz von Funkzellenabfragen und für die Erfüllung der Benachrichtigungs- und Löschungspflichten einzelfallbezogen zu dokumentieren sind. Außerdem sollten Vorgaben für die Umsetzung der Kennzeichnung gemacht werden. Schließlich wird die Einführung einer regelmäßigen Berichtspflicht gegenüber dem Abgeordnetenhaus von Berlin und dem Berliner Beauftragten für Datenschutz und Informationsfreiheit empfohlen.

- Auch sollte das Land Berlin sich für eine Präzisierung der strafprozessualen Regelungen zur Funkzellenabfrage einsetzen. Zumindest muss diese weitreichende Maßnahme auf den Straftatenkatalog des $\$ 100$ a StPO beschränkt werden. Die Grundsätze der Zweckbindung und Verhältnismäßigkeit müssen für die Funkzellenabfrage konkretisiert und stärker zur Geltung gebracht werden. Der Bundesgesetzgeber muss durch eine Änderung der Strafprozessordnung sicherstellen, dass die Funkzellenabfrage von einer Routinemaßnahme wieder zur ultima ratio wird.

Dr. Alexander Dix, LL.M. Berliner Beauftragter für Datenschutz und Informationsfreiheit 